\title{
NOUVELLE
}

\section{Les radeaux de la mort Quand les radeaux lipidiques régulent les récepteurs à dépendance}

Céline Furne, Patrick Mehlen
Apoptose, Cancer et Dévelopement, Équipe labellisée «La Ligue », CNRS FRE2870, Centre Léon Bérard, 69008 Lyon, France. mehlen@lyon.fnclcc.fr
> Il est classiquement admis qu'un récepteur est uniquement actif lorsqu'il interagit avec son ligand. La famille des récepteurs dits «à dépendance » est l'exception qui confirme la règle. En effet, en présence de ligand, ces récepteurs induisent des signaux de prolifération, différenciation ou migration, mais ils sont

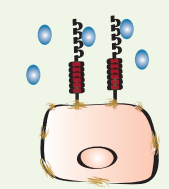

Transformation cellulaire
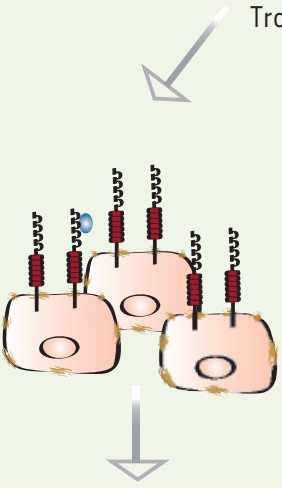

Apoptose induite par DCC

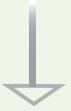

Suppression des cellules tumorales

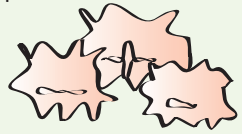
ligand puisqu'ils induisent la mort de la cellule par apoptose. Ces récepteurs sont donc dits «à dépendance » car une cellule qui les exprime devient dépendante de la présence du ligand pour survivre. Cette famille émergente compte aujourd'hui une dizaine de également actifs en absence de leur

membres: P75NTR, DCC, les récepteurs UNC5H1, UNC5H2 et UNC5H3, RET, le récepteur aux androgènes, les intégrines $\alpha_{V} \beta_{3}$ et $\alpha_{5} \beta_{1}$, Patched et Néogenine [1].

Le récepteur DCC (deleted in colorectal cancer) est un récepteur transmembranaire initialement décrit comme un potentiel suppresseur de tumeurs car son expression est perdue dans $70 \%$ des cancers colorectaux ainsi que dans de nombreux autres cancers. Si l'activité suppresseur de tumeurs de DCC n'est pas encore formellement démontrée, il semblerait que DCC prévienne l'échappement tumoral en éliminant par apoptose les cellules cancéreuses. En effet, les cellules tumorales prolifèrent intensément dans un environnement où la concentration en nétrine-l reste constante, les cellules seraient donc en déficit de nétrine-1 et DCC déclencherait leur apoptose. De la même manière, une cellule tumorale métastatique qui quitterait le site primaire de tumorigenèse pour envahir d'autres tissus perdrait la nétrine-l et entrerait alors en apoptose. Ainsi, une perte d'expression de DCC, suite à une perte d'hétérozygotie ou à la méthylation du promoteur, serait un avantage sélectif qui rendrait les cellules résistantes à l'absence de nétrine-1. De même, une surexpression de nétrine-1 par les cellules environnantes ou par les cellules tumorales elles-mêmes permettrait aux cellules cancéreuses de proliférer ou d'envahir d'autres tissus. Ainsi, des souris transgéniques, dont la mort induite par DCC est inhibée par une surexpression de nétrine-1, sont prédisposées au développement de tumeurs colorectales [2].

Le récepteur DCC n'est pas seulement impliqué dans les processus tumoraux puisqu'au cours
Figure 1. Le récepteur DCC. II s'agit d'un suppresseur de tumeurs présomptif qui contrôlerait l'échappement tumoral en induisant l'apoptose des cellules qui se retrouvent déficitaires en nétrine-l car elles ont proliféré ou migré vers d'autres tissus dépourvus de nétrine-1. La perte d'expression du récepteur et la surexpression ou l'expression autocrine de nétrine-l seraient des avantages sélectifs pour le développement tumoral. Une altération de la localisation de DCC dans les radeaux lipidiques, à la suite d'une modification des lipides membranaires ou de la palmitoylation du récepteur, pourrait conduire à une perte de l'activité pro-apoptotique de DCC. 
du développement, via l'interaction avec son ligand, la nétrine-1, il contrôle le guidage axonal et la migration neuronale [3]. De récentes études ont montré que les radeaux lipidiques modulent le guidage axonal assuré par le couple $\mathrm{DCC} /$ nétrine-1 $[4,5]$. Les radeaux lipidiques sont des microdomaines membranaires particulièrement riches en phospholipides, sphingolipides et cholestérol. Ils présentent des compositions protéiques particulières et participent à de nombreux processus cellulaires comme la transduction du signal, l'endocytose et l'exocytose, ou encore l'apoptose.

Un article émanant d'un travail collaboratif entre le groupe de A.0. Hueber et le nôtre révèle que la localisation de DCC dans les radeaux lipidiques est cruciale pour l'induction de l'apoptose [6]. En effet, le récepteur DCC est partiellement associé aux radeaux lipidiques. Cette localisation ne dépend pas de la nétrine- 1 mais de l'ajout post-traductionnel d'un acide palmitique sur une cystéine localisée à l'extrémité du domaine transmembranaire du récepteur. Si cette cystéine est remplacée par une valine, DCC n'est plus palmitoylé et n'est donc plus associé aux radeaux lipidiques. Cette localisation de DCC dans les radeaux lipidiques est importante car une altération des rafts par dégradation du cholestérol ou des sphingolipides réduit fortement son activité pro-apoptotique, aussi bien dans des cellules tranfectées que dans des neurones spinaux qui expriment DCC de manière endogène. De manière plus spécifique, le mutant de DCC qui n'est plus palmitoylé, et n'est donc plus localisé dans les rafts perd son activité de mort.

Les radeaux lipidiques modulent principalement la transduction du signal en établissant des plateformes protéiques autour des récepteurs membranaires. $\mathrm{Si}$ la signalisation apoptotique de DCC reste peu connue, il semblerait qu'en l'absence de nétrine-1, pour induire la mort, DCC interagisse avec la caspase 9, une protéase centrale de l'apoptose, capable d'engendrer, par clivage, une activation en cascade d'autres caspases et ainsi de conduire à la mort de la cellule. Cette interaction avec la caspase- 9 , indispensable à l'activité apoptotique de DCC, est abolie lorsque DCC n'est plus palmitoylé ou que la membrane cellulaire est déplétée en cholestérol. La localisation de DCC au sein des radeaux lipidiques serait donc importante pour son activité pro-apoptotique car elle permettrait la formation d'un complexe d'activation des caspases.

À partir de ce travail de signalisation très académique, notre intérêt est aujourd'hui de resituer l'importance de la localisation de DCC dans les radeaux lipidiques dans son activité de suppresseur de tumeurs. En effet, nous proposons que cette activité est liée à sa fonction pro-apoptotique [1-3]. Comme cela a été décrit précédemment, une inhibition de cette activité par perte d'expression de DCC ou par surexpression de nétrine-1 serait alors un avantage sélectif pour les cellules tumorales. Notre étude propose alors qu'une altération de la localisation membranaire de DCC pourrait également conduire à la perte d'activité apoptotique de DCC dans des cellules tumorales. $\diamond$

The deadly rafts: when lipid rafts regulate dependence receptors

\section{RÉFÉRENCES}

1. Mehlen P, Thibert C. Dependence receptors : between life and death. Cell Mol Life Sci 2004 ; 61 : 1854-66.

2. Mazelin L, Bernet A, Bonod-Bidaud C, et al. Netrin1 controls colorectal tumorigenesis by regulating apoptosis. Nature $2004 ; 431: 80-4$.

3. Mehlen P, Furne C. Netrin-1: when a neuronal guidance cue turns out to be a regulator of tumorigenesis. Cell Mol Life Sci $2005 ; 62$ : 2599-616.

4. Herincs Z, Corset V, Cahuzac N, et al. DCC association with lipid rafts is required for netrin-1-mediated axon guidance. J Cell Sci 2005 ; 118 : 1687-92.

5. Guirland C, Suzuki S, Kojima M, et al. Lipid rafts mediate chemotropic guidance of nerve growth cones. Neuron $2004 ; 42: 51-62$.

6. Furne $C$, Corset $V$, Herincs $Z$, et al. The dependence receptor DCC requires lipid raft localization for cell death signaling. Proc Natl Acad Sci USA 2006 ; 103 : 4128-33.

\section{NOUVELle}

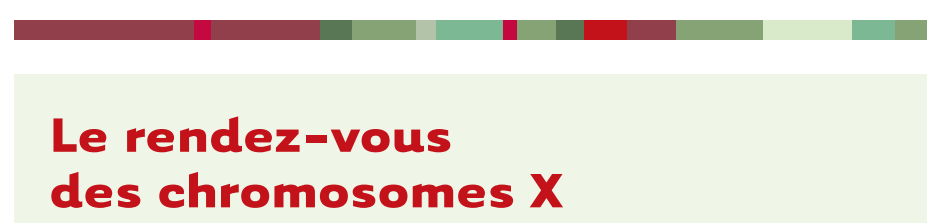

Sandrine Augui, Edith Heard

> Chez les mammifères, la divergence entre le chromosome $X$, riche en gènes, et le chromosome $y$, particulièrement pauvre, engendre un déséquilibre génique entre la femelle XX et le mâle XY $(\rightarrow)$. Ce déséquilibre est compensé très tôt au cours du développe-

$(\rightarrow) \mathrm{m} / \mathrm{s} 2006$, $n^{\circ} 11$, p. 926

ment embryonnaire par l'inactivation chez la femelle d'un des deux chromoso- mes $X$. La quasi totalité des gènes portés par ce chromosome sont alors éteints. La mise en place de l'inactivation est contrôlée par un locus unique, appelé centre d'inactivation du chromosome $X$ (ou $X i c$ ), qui réunit sur plusieurs centaines de kilobases l'ensemble des acteurs connus du processus. Parmi eux, le gène Xist et son antisens Tsix. Xist, gène clé de l'inactivation, code un ARN non tra-
Equipe Épigenèse

et Développement des mammifères,

CNRS UMR 218, Institut Curie,

26, rue d'Ulm, 75248 Paris Cedex 06, France.

Edith.Heard@curie.fr

duit qui recouvre le chromosome $X$ à inactiver et induit son extinction par des mécanismes encore inconnus à ce jour; Tsix, quant à lui, est impliqué dans la régulation de Xist [1].

Dans l'embryon, dont proviennent les cellules souches embryonnaires (ou cellules ES), l'inactivation touche aléatoirement l'un des deux chromosomes X. Aucun $X$ n'est donc prédestiné à être inactivé. 